González Cabanach, R.; Souto-Gestal, A.; González-Doniz, L. y Franco Taboada, V. (2018). Perfiles de afrontamiento y estrés académico en estudiantes universitarios. Revista de Investigación Educativa, 36(2), 421-433.

DOI: http://dx.doi.org/10.6018/rie.36.2.290901

\title{
Perfiles de afrontamiento y estrés académico en estudiantes universitarios
}

\section{Profiles of coping and academic stress among university students}

\author{
Ramón González Cabanach,Antonio Souto-Gestal, \\ Luz González-Doniz y Victoria Franco Taboada \\ Universidade da Coruña. Grupo de investigación en Intervención Psicosocial y Rehabilitación Funcional, \\ Facultad de Fisioterapia, Oza, I 507 I A Coruña, Spain.
}

\begin{abstract}
Resumen
Introducción: los efectos del estrés académico se encuentran mediados por las estrategias de afrontamiento de los estudiantes. Sin embargo, existe poca evidencia acerca de la asociación entre los diferentes perfiles de afrontamiento y las respuestas psicofisiológicas de estrés. El objetivo de la presente investigación es analizar la variabilidad de las respuestas psicofisiológicas de estrés en función del perfil de afrontamiento activo que presenta el estudiante universitario. Método: participaron en el estudio 1195 estudiantes universitarios, el 71\% mujeres, con una edad media de 21.57 años (DT=3.63). Se administró la escala de afrontamiento de estresores (ACEA) y la escala de respuestas de estrés (RCEA) del cuestionario de estrés académico. Resultados: un quick cluster clasificó a los estudiantes en 4 grupos. Dos de los grupos mostraron un alto grado de afrontamiento activo (afrontamiento activo [AA] y afrontamiento activo y social [AAS]), mientras que los otros dos reportaron niveles bajos (afrontamiento social [AS] y bajo afrontamiento activo y social [BAS]). El ANOVA mostró que los estudiantes que emplean estrategias de afrontamiento activo ( $A A$ y AAS) informan de menores respuestas psicofisiológicas de estrés en comparación aquellos con bajo nivel de afrontamiento activo ( $A S$ y BAS), tanto físicas (agotamiento físico y alteraciones del sueño), como conductuales (irascibilidad) y cognitivas (intrusión de pensamientos negativos). La función mediadora del apoyo social se muestra
\end{abstract}

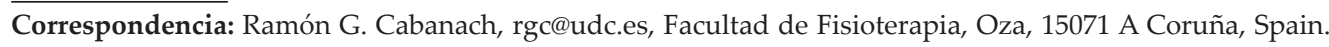


importante únicamente entre los grupos con niveles bajos de afrontamiento activo (AS y BAS). Conclusión: los programas de prevención e intervención en el estrés académico deberían tener en cuenta estas diferencias en su diseño e implementación.

Palabras clave: estrés; afrontamiento; apoyo social; estudiantes universitarios.

\begin{abstract}
Introduction: Effects of academic stress are mediated by the students coping strategies. However, there is little evidence on the association between different profiles of coping and stress psychophysiological responses. The aim of this study is to analyze the variability of psychophysiological stress responses in relation to the profile of active coping that students show. Method: 1195 Spanish university students (71\% females) with a mean age of 21.57 $(S D=3.63)$ participated in the study. Self-administered instruments stress coping scale ( $A C E A)$ and stress response scale (ACEA) were administered. Results: participants were classified into 4 groups by a quick cluster. Two groups showed high levels of active coping (active coping $[A C]$ and social and active coping [SAC]), whereas other two groups reported low levels (social coping [SC] and low social and active coping [LSA]). One-way ANOVA showed that students classified within groups of high active coping ( $A C$ and $S A C)$ exhibited lower scores in psychophysiological responses of stress (physical exhaustion, sleep disturbances, irascibility and negative cognitions intrusion) than those students classified within groups of low active coping (SC and LSA). Additionally, the mediating role of social support result relevant only among students with low level of active coping (SC and SAC). In conclusion, academic stress prevention and intervention programs should take into account the peculiarity of this subset in its design and implementation.
\end{abstract}

Keywords: stress; coping; social support; university students.

\title{
Introducción
}

El modelo transaccional caracteriza el afrontamiento como un proceso dinámico, de carácter cognitivo y conductual, dirigido a activar los recursos necesarios para hacer frente a las demandas externas o internas. Se trata de un proceso esencial para que la persona pueda controlar el estrés, regulando las emociones suscitadas y evitando la aparición de un estado de desequilibrio psicofisiológico. En esencia, el afrontamiento implica la puesta en marcha de un conjunto de procesos de autorregulación que interactúan entre sí para hacer frente a los efectos desencadenados por la percepción de estrés (Compas, Connor-Smith, Saltzman, Thomsen \& Wadsworth, 2001).

Una de las cuestiones claves en la investigación actual sobre el afrontamiento es la de su eficacia. En concreto, interesa conocer qué tipo de afrontamiento resulta más eficaz y qué criterios nos sirven para evaluar esta eficacia.

En relación con el primero de los aspectos, aunque se ha considerado tradicionalmente que existen formas de afrontamiento más efectivas que otras para ayudar a los individuos a mejorar su estado de bienestar, se trata de una temática en discusión. Así, por ejemplo, Somerfield y McCrae (2000) han señalado la dificultad de determinar la eficacia mayor o menor de un tipo de afrontamiento u otro, ya que la idoneidad 
de una estrategia depende en gran medida del contexto concreto en el que se aplica, y lo que es adecuado en una situación no necesariamente lo hace idóneo para otra.

Pero a pesar de la complejidad de esta cuestión, la investigación ha aportado alguna información relevante para la búsqueda de soluciones. En efecto, diversos estudios han argumentado la mayor eficacia de las estrategias centradas en el problema (afrontamiento activo), que se han asociado con un mayor bienestar (Cornejo \& Lucero, 2005; Fierro \& Jiménez, 2002).

En una revisión sobre esta temática Fernández y Polo (2011) afirman que el bienestar del individuo representa un indicador muy relevante para valorar la eficacia de una estrategia o estilo de afrontamiento. En este sentido, el empleo de este tipo de estrategias de afrontamiento, dirigidas a solucionar problemas, no sólo reduce los efectos o resultados del estrés, sino también las consecuencias (o efectos a más largo plazo) tales como los síntomas depresivos, fóbicos y las sensaciones de malestar psicológico. Por el contrario, los estudiantes que utilizan en menor medida estas estrategias o lo hacen de forma inadecuada, y recurren más a la evitación y la descarga emocional, presentan un mayor nivel de malestar y síntomas de somatización, depresión y ansiedad. En concreto, las estrategias de evitación conductuales (el consumo de alcohol o drogas) se asociaron con un mayor malestar psicológico y mayor presencia de depresión.

Sin embargo, cabe señalar que la mayor parte de trabajos que analiza el efecto de las estrategias de afrontamiento han buscado su relación con el desempeño académico de los estudiantes o con la percepción de estresores (e.g., de la Fuente et al., 2017; Thomas, Cassady \& Heller, 2017). De este modo, Fornés-Vives, Garcia-Banda, Frias-Navarro y Rosales-Viladrich (2016), pusieron de manifiesto como el empleo de estrategias de afrontamiento dirigidas a la solución de problemas se acompañaba de una reducción en la percepción de estresores en una muestra de estudiantes de enfermería españoles.

Para Cabanach, Valle, Rodríguez, Piñeiro y Freire (2010), las principales estrategias de afrontamiento activo se podrían clasificar en tres grandes grupos. En primer lugar, la reevaluación positiva incluye todas aquellas acciones dirigidas a crear un nuevo significado de las situaciones estresantes, realzando su parte positiva y minimizando los aspectos negativos de la misma. Por otro lado, la búsqueda de apoyo social aglutinaría aquellas conductas que poseen una doble función, por un lado la búsqueda de apoyo social instrumental al problema (búsqueda de información adicional que contribuya a la resolución del problema) y por otro, la búsqueda de apoyo social emocional, centrada en la búsqueda de comprensión y alivio emocional por parte de otros. Por último, todas aquellas acciones que se fundamentan la modificación de la situación problemática a partir de una aproximación analítica y racional a la misma, se incluirían dentro de las estrategias de planificación estratégica de recursos personales.

En relación con los criterios de evaluación de la eficacia del afrontamiento, se han propuesto varios para comprobar la eficacia de un afrontamiento por sus resultados y consecuencias, tales como reducir el malestar psicológico, resolver el problema, conservar la autoestima o la percepción subjetiva de eficacia, entre otros (Zeidner \& Saklofske, 1996). Probablemente los dos criterios que parecen indicar una mayor eficacia son los del bienestar del individuo (Denovan \& Macaskill, 2017; Freire, 
Ferradás, Valle, Núñez \& Vallejo, 2016) y la reducción del malestar, expresada en la reducción de la sintomatología psicofisiológica de naturaleza cognitiva, emocional, conductual y física (Cabanach, Valle et al., 2010; Pozos-Radillo, Preciado-Serrano, Plascencia-Campos, Valdez-López \& Morales-Fernández, 2016).

En otro estudio reciente, Freire et al. (2016), empleando una metodología similar en un contexto comparable al del presente trabajo, relacionaron un perfil de mayor bienestar psicológico en estudiantes con el empleo de estrategias de afrontamiento activo basadas en reevaluación positiva, búsqueda de apoyo social y planificación estratégica. Sin embargo, parecería también razonable que los perfiles de afrontamiento que caracterizan al estudiante universitario puedan condicionar una mayor o menor vulnerabilidad de éste frente al estrés académico y, por extensión, amenazar su bienestar. De este modo, el objetivo de la presente investigación se centra en analizar la variabilidad de las respuestas psicofisiológicas de estrés en función del perfil de afrontamiento activo que presenta el estudiante universitario.

\section{Método}

\section{Objetivos}

En función de las aportaciones teóricas revisadas, en nuestra investigación nos hemos planteado un objetivo fundamental, que resume la problemática analizada anteriormente: constatar si el afrontamiento activo es el más eficaz en la reducción del estrés académico. Ello lo hemos medido teniendo en cuenta la mayor reducción de sus manifestaciones psicofisiológicas. Para evaluar este afrontamiento hemos utilizado una estrategia de tipo cognitivo (la reevaluación positiva) y otra de afrontamiento conductual (la planificación y gestión de recursos). También hemos medido el afrontamiento social (la búsqueda de apoyo social), como exponente de estrategia mixta, con la intención de analizar las diferencias con las otras dos estrategias en la reducción del malestar psicofisiológico.

\section{Población y Muestra}

Participaron en el estudio 1195 estudiantes de diferentes titulaciones de la Universidad de A Coruña. En concreto, de titulaciones de los ámbitos de conocimiento de las ciencias sociales y jurídicas (Administración de Empresas y Derecho, Educación Social y Económicas), de estudios técnicos (Arquitectura e Ingeniería de Caminos, Canales y Puertos) y de ciencias de la salud (Logopedia y Fisioterapia).

Las estadísticas de los últimos años describen la población de estudiantes universitarios que cursan titulaciones de grado en el Campus de A Coruña entorno a los 16 mil estudiantes. La selección de la muestra se llevó a cabo empleando un muestreo por conglomerados de una única etapa, respetando el agrupamiento natural por cursos de los estudiantes participantes. Se considera como conglomerado un grupo establecido de estudiantes por clase, con una participación voluntaria en la recogida de datos por medio de cuestionarios, garantizando en todo momento el anonimato en el tratamiento de los datos. Participaron aquellos centros que, previo contacto por parte del equipo de 
investigación, cedieron sus espacios para la realización de la investigación con aquellos estudiantes que acudieron a clase y voluntariamente accedieron a cumplimentar el cuestionario (muestra aceptante).

De estos 1195 estudiantes, 825 eran mujeres (71.4\%) y 331 hombres (28.6\%), con edades comprendidas entre 18 y 47 años, con una media de edad de 21.57 años (DE=3.63).

\section{Instrumento}

\section{Escala de Afrontamiento (ACEA) del Cuestionario de Estrés Académico}

El afrontamiento fue medido mediante la escala de afrontamiento (ACEA), que forma parte del "Cuestionario de Estrés Académico" elaborado por Cabanach, Valle et al. (2010). Se compone de un total de 23 ítems que tratan de medir en qué grado el estudiante emplea determinadas estrategias de afrontamiento cognitivas, conductuales y sociales para dar respuesta al estrés académico. Las respuestas a cada uno de los ítems se realizan sobre una escala de estimación tipo Likert de 5 puntos, en la que el estudiante indica la frecuencia con la que utiliza determinadas estrategias de afrontamiento, desde "Nunca" (1) hasta "Siempre" (5). Agrupa tres factores que, con los datos de la presente muestra, explican el $60.98 \%$ de la varianza total, un valor ligeramente superior al obtenido en la validación original de este instrumento (Cabanach, Valle et al., 2010). Estos tres factores son los de reevaluación positiva, búsqueda de apoyo social y planificación y gestión de recursos personales.

En estudios precedentes (e.g., Cabanach, Cervantes, González-Doniz \& Freire, 2010) esta escala mostró una buena consistencia interna, con un valor a de Cronbach para el total de la escala de .89 , con valores comprendidos entre .85 y .88 para los tres factores. Los valores de consistencia interna obtenidos con los datos del presente estudio son también excelentes, con un valor a igual a .92 para el conjunto de la escala, y de entre .86 y .90 para cada uno de los tres factores señalados.

\section{Escala de Respuesta (RCEA) del Cuestionario de Estrés Académico}

Está integrada por 22 ítems que miden síntomas vinculados a los efectos fisiológicos, cognitivos, conductuales y afectivos relacionados con el estrés académico. Las respuestas a cada uno de los ítems se realizan sobre una escala tipo Likert de 5 puntos, en la que el estudiante indica la frecuencia con la que experimenta determinados síntomas, desde "Nunca" (1) hasta "Siempre" (5).

Estos ítems se agrupan en cuatro factores, que explican el 68.43\% de la varianza. Estos cuatro factores son los siguientes: agotamiento físico, dificultades del sueño, irascibilidad y pensamientos negativos.

En estudios precedentes (e.g., Cabanach, Cervantes et al., 2010) esta escala mostró poseer una excelente consistencia interna, con un valor a de Cronbach para el total de la escala de .93 , con valores comprendidos entre .80 y .88 para los factores descritos. Los valores de consistencia interna obtenidos con los datos del presente estudio se muestran igualmente excelentes, con un valor a igual a .93 para el conjunto de la escala, y de entre .85 y .93 para cada uno de los cuatro factores descritos. 


\section{Procedimiento de recogida y análisis de datos}

El estudio se llevó a cabo siguiendo un diseño descriptivo mediante encuesta de corte transversal (Montero \& León, 2002). Se procedió a la recogida de los datos relativos a las variables objeto de análisis en cada uno de los centros a los que asistían los estudiantes que participaron en la investigación. La recogida de información fue realizada mediante la técnica de cuestionario. Estos fueron aplicados, individualmente, sin límite de tiempo y en un único momento temporal, por personal especializado que colaboró en el estudio. A los participantes se les recalcó la importancia de responder con sinceridad a todas las cuestiones planteadas. Asimismo, se les informó del carácter totalmente confidencial de dicha información.

Con el propósito de identificar los perfiles de afrontamiento de los estudiantes de la muestra utilizamos el análisis de conglomerados (método quick cluster analysis). Se trata de un método no jerárquico y de reasignación. Este análisis de conglomerados se considera un procedimiento adecuado para el establecimiento de patrones en una muestra amplia de sujetos (Hair, Anderson, Tatham \& Black, 1999; Pastor, Barron, Miller \& Davis, 2007).

Los perfiles de afrontamiento se han definido a partir de las diferentes combinaciones de los tres tipos señalados anteriormente. Para eliminar el efecto debido a las diferencias en la medida de estas dimensiones (el número de ítems de cada una de los factores es diferente), se ha realizado el análisis de conglomerados después de haber estandarizado las puntuaciones directas. El criterio seguido en la elección del número de conglomerados fue el de maximizar las diferencias inter-clusters con el fin de conseguir establecer el mayor número posible de grupos con diferentes combinaciones de dimensiones de afrontamiento.

Para conocer las posibles diferencias entre los perfiles respecto a las manifestaciones psicofisiológicas de estrés, hemos utilizado el análisis de varianza (ANOVA) tras comprobar el supuesto de normalidad a través de la prueba de Kolmogorov-Smirnov. Se empleó Scheffé como prueba de contraste post hoc en aquellos casos en los que resultó posible asumir homogeneidad de varianzas, y Games-Howell cuando no fue posible asumir el supuesto de homocedasticidad mediante la prueba de Levene. Se asumió un nivel de significación de .05 y adicionalmente se calculó la $d$ de Cohen como medida del tamaño del efecto.

\section{Resultados}

Los resultados ponen de manifiesto la existencia de cuatro perfiles claramente diferenciados (ver figura 1). Un primer perfil, que podríamos denominar como de afrontamiento activo (AA), que presenta altas puntuaciones en las dos estrategias de afrontamiento activo (reevaluación positiva y planificación y gestión de recursos) y bajas puntuaciones en afrontamiento social (búsqueda de apoyo social). Un segundo perfil, que denominaremos de afrontamiento social (AS) (bajo afrontamiento activo y alto afrontamiento social). Un tercer perfil, que hemos denominado de bajo afrontamiento activo y social (BAS), que presenta puntuaciones muy bajas en las tres estrategias de afrontamiento medidas. Un cuarto perfil, al que hemos denominado como afrontamiento activo y social (AAS), con altas puntuaciones en todas las estrategias de afrontamiento que han sido medidas (las dos de afrontamiento activo y la de afrontamiento social). 


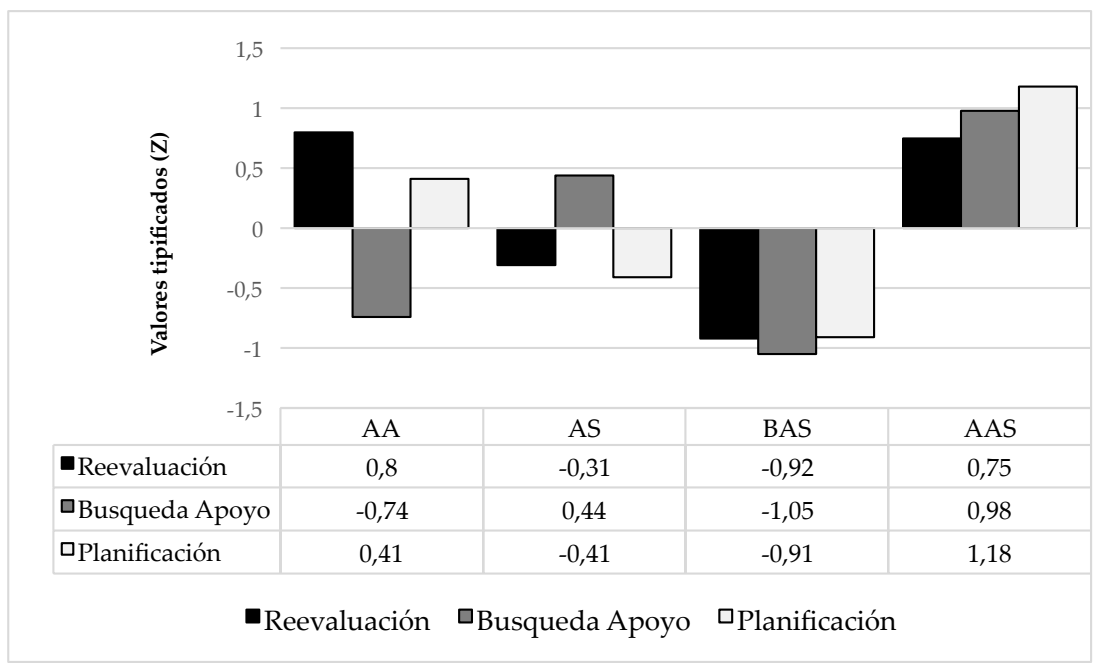

Figura 1. Perfiles de afrontamiento identificados por el análisis clúster.

El número de estudiantes que integran los perfiles BAS y ASS es bastante similar (289 y 290 estudiantes respectivamente), mientras que el primero de los perfiles (AA) agrupa a 215 estudiantes. El perfil que reúne más estudiantes es el segundo (AS), con 401 sujetos.

En cuanto a la relación entre perfiles de afrontamiento y manifestaciones psicofisiológicas del estrés (ver tabla 1), que es el objetivo de este estudio, vemos que es siempre el grupo AA (perfil de afrontamiento activo) el que obtiene puntuaciones más bajas en todas las respuestas de estrés medidas, tanto las de tipo físico como en las conductuales y cognitivas.

En comparación con el grupo BAS, los estudiantes del grupo AA muestran diferencias estadísticamente significativas en las dos respuestas físicas medidas (agotamiento físico y dificultades del sueño), así como en la conductual y en la cognitiva. Los tamaños del efecto detectados se encuentran en todos los casos por encima de .41, alcanzando un valor de .62 para la dimensión pensamientos negativos.

La comparación entre los grupos AA y AS (bajo afrontamiento activo y alto afrontamiento social) arroja diferencias significativas para una de las manifestaciones fisiológicas (agotamiento físico) y para la de tipo cognitivo (pensamientos negativos). En ambos casos la magnitud de las diferencias se aproxima a .30.

Sin embargo, no se han encontrado diferencias significativas entre los dos grupos con elevado afrontamiento activo (AA y AAS), mostrando el primero de ellos valores ligeramente más reducidos en todas las respuestas psicofisiológicas.

Por su parte, el grupo caracterizado por el afrontamiento activo y social (AAS) también presenta diferencias significativas con respecto al grupo BAS, mostrando niveles inferiores para las cuatro respuestas medidas, tanto físicas como psicológicas (conductuales y cognitivas). No obstante, dichas diferencias desaparecen cuando son comparados con el grupo de alto afrontamiento social (AS). 
Tabla 1

Descriptivos y valores significación estadística para las diferencias de respuestas de estrés entre los diferentes perfiles de afrontamiento.

\begin{tabular}{|c|c|c|c|c|c|c|}
\hline Respuesta de estrés & Perfil & $N$ & $M$ & $D E$ & $F$ & $p$ \\
\hline \multirow{4}{*}{ AGOTAMIENTO FÍSICO } & $\mathrm{AA}$ & 215 & 2.73 & 1.05 & \multirow{4}{*}{9.13} & \multirow{4}{*}{$<.001$} \\
\hline & AS & 401 & 3.02 & 1.07 & & \\
\hline & BAS & 289 & 3.21 & 1.04 & & \\
\hline & AAS & 290 & 2.89 & 1.06 & & \\
\hline \multirow{4}{*}{$\begin{array}{l}\text { ALTERACIONES DEL } \\
\text { SUEÑO }\end{array}$} & AA & 215 & 1.90 & .90 & \multirow{4}{*}{7.92} & \multirow{4}{*}{$<.001$} \\
\hline & AS & 401 & 2.08 & .98 & & \\
\hline & BAS & 289 & 2.28 & .97 & & \\
\hline & AAS & 290 & 1.97 & .97 & & \\
\hline \multirow{4}{*}{ IRASCIBILIDAD } & AA & 215 & 1.99 & .90 & \multirow{4}{*}{10.74} & \multirow{4}{*}{$<.001$} \\
\hline & AS & 401 & 2.18 & 1.02 & & \\
\hline & BAS & 289 & 2.43 & 1.05 & & \\
\hline & AAS & 290 & 2.03 & 1.04 & & \\
\hline \multirow{4}{*}{$\begin{array}{l}\text { PENSAMIENTOS } \\
\text { NEGATIVOS }\end{array}$} & AA & 215 & 1.89 & .83 & \multirow{4}{*}{18.28} & \multirow{4}{*}{$<.001$} \\
\hline & AS & 401 & 2.16 & 1.00 & & \\
\hline & BAS & 289 & 2.51 & 1.15 & & \\
\hline & AAS & 290 & 2.00 & 1.052 & & \\
\hline
\end{tabular}

Tabla 2

Valores significación estadística para las comparaciones post hoc

\begin{tabular}{|c|c|c|c|c|c|c|c|}
\hline Respuesta & Post hoc & $\mathrm{p}$ & $d$ & Respuesta & Post hoc & p & $d$ \\
\hline \multirow{6}{*}{$\begin{array}{l}\text { Agotamiento } \\
\text { físico }\left(^{*}\right)\end{array}$} & AA-AS & .019 & .27 & \multirow{6}{*}{ Irascibilidad $\left(^{*}\right)$} & AA-AS & .146 & .20 \\
\hline & AA-BAS & $<.001$ & .46 & & AA-BAS & $<.001$ & .45 \\
\hline & AA-AAS & .419 & .15 & & AA-AAS & .965 & .04 \\
\hline & AS-BAS & .143 & .18 & & AS-BAS & .017 & .24 \\
\hline & AS-AAS & .518 & .12 & & AS-AAS & .290 & .15 \\
\hline & BAS-AAS & .006 & .30 & & BAS-AAS & $<.001$ & .38 \\
\hline \multirow{6}{*}{$\begin{array}{l}\text { Alteraciones } \\
\text { del sueño }\left(^{*}\right)\end{array}$} & AA-AS & .203 & .19 & \multirow{6}{*}{$\begin{array}{l}\text { Pensamientos } \\
\text { negativos }\left({ }^{* *}\right)\end{array}$} & AA-AS & .022 & .29 \\
\hline & AA-BAS & $<.001$ & .41 & & AA-BAS & $<.001$ & .62 \\
\hline & AA-AAS & .898 & .08 & & AA-AAS & .736 & .12 \\
\hline & AS-BAS & .055 & .21 & & AS-BAS & $<.001$ & .32 \\
\hline & AS-AAS & .547 & .11 & & AS-AAS & .225 & .16 \\
\hline & BAS-AAS & .002 & .32 & & BAS-AAS & $<.001$ & .46 \\
\hline
\end{tabular}

Nota: * Scheffé; ** Games-Howell. 
Finalmente, entre los grupos de bajo afrontamiento activo, encontramos que el grupo BAS presenta de forma estadísticamente significativa valores superiores en las dos respuestas de estrés psicológicas medidas (irascibilidad y pensamientos negativos), en comparación con el grupo AS (afrontamiento social).

En resumen, son los grupos con un perfil de afrontamiento activo (AA y AAS) los que presentan menores manifestaciones psicofisiológicas de estrés. Puede afirmarse, por tanto, que aquellos estudiantes con un afrontamiento activo de las situaciones de estrés presentan menores manifestaciones psicofisiológicas de estrés.

Por su parte, los grupos que usan menos las estrategias de afrontamiento activo (AS y BAS), son los que se ven más afectados por las manifestaciones psicofisiológicas de estrés. Dicho de otra manera, los que más estrés manifiestan. Ello refuerza la afirmación de que son las estrategias de afrontamiento activo las más adaptativas ante las situaciones de estrés académico.

\section{Discusión y conclusiones}

La estrategia de afrontamiento búsqueda de apoyo social caracteriza a más de la mitad de la muestra, a través de los grupos AS y ASS. Teniendo en cuenta estudios precedentes, es posible que ello sea debido a la existencia de una presencia superior de mujeres en la muestra, que se caracterizan por un uso claramente diferencial con respecto a los hombres de las estrategias de afrontamiento social (en concreto, la búsqueda de apoyo social) (Cabanach, González-Millán \& Freire, 2009). Futuros estudios deberían analizar la posible existencia de patrones perfiles de afrontamiento diferentes en función del género.

Nuestros resultados permiten afirmar claramente que las estrategias de afrontamiento activo son las más eficaces para enfrentarse a las situaciones académicas de estrés, reduciendo el impacto estresante de las mismas, respondiendo así de forma afirmativa a la pregunta que nos hacíamos en el título de este trabajo. El uso de las estrategias de afrontamiento activo está significativamente asociado con menores manifestaciones psicofisiológicas de estrés. La utilización de estas estrategias parece estar claramente relacionada con una menor vulnerabilidad al estrés por parte de estos estudiantes universitarios. Por tanto, estos estudiantes son más capaces de enfrentar con éxito las situaciones académicas de estrés, manteniendo el equilibrio físico y mental necesario para adaptarse adecuadamente al contexto académico, progresar en sus estudios y, probablemente, rendir mejor. Sin embargo, hay un dato al que conviene aludir: esta adaptación a las situaciones de estrés es superior en los estudiantes que usan más las estrategias consideradas de afrontamiento activo, siendo inferior -aunque la diferencia no alcance niveles de significación- en aquéllos que usan en mayor medida el afrontamiento social (grupo ASS).

Por otra parte, los estudiantes que hacen un menor uso de las estrategias de afrontamiento activo son los más vulnerables al estrés académico y los que manifiestan un mayor malestar. Es posible que ello tenga alguna relación con el hecho de que el estudiante percibe muchas de las situaciones académicas como controlables -al menos, las más significativas en su desempeño académico-, es decir, situaciones en las que sabemos de forma consciente que algo podemos hacer, considerando, por tanto, que 
puede enfrentarse a ellas, empleando mecanismos de autorregulación que actúen positivamente sobre sus efectos psicofisiológicos y le permitan mantener su equilibrio y su adaptación. Las personas más resistentes parecen dotarse y desarrollar maneras de afrontar los problemas y dificultades de la vida de una forma constructiva. Estos modos de afrontamiento se adaptan a la tipología de los acontecimientos y son realistas.

Dentro de estos grupos con un bajo nivel de afrontamiento activo, podemos señalar el impacto diferencial del uso de las estrategias de afrontamiento social. En efecto, el grupo AS, que presenta niveles más elevados del uso de estas estrategias, manifiesta menores efectos psicofisiológicos del estrés. Ello es claramente evidente en la respuesta cognitiva (pensamientos negativos), de notable impacto en el estrés. Probablemente, el menor uso de las estrategias de afrontamiento activo (reevaluación positiva y planificación) esté compensado parcialmente con el uso de las estrategias de afrontamiento social.

Como afirman Johnson y Johnson (2002), dotarse de estas habilidades de afrontamiento es esencial para que los estudiantes puedan adaptarse saludablemente y promover un sentimiento de bienestar. Distintos estudios (Carver, Scheier \& Pozo, 1992; Thomas et al., 2017; Zeidner \& Saklofske, 1996) avalan la idea de que su grado de adaptación y su funcionamiento vital y académico mejoran cuando realizan una valoración positiva de los acontecimientos y recurren a estrategias de resolución de problemas. Existe consenso entre los estudiosos en que la validez de una estrategia de afrontamiento es refrendada si favorece el estado de bienestar de la persona, entendiendo éste en su más amplio espectro: se estaría hablando de bienestar físico, psicológico y social (Freire et al., 2016; Parsons, Frydenberg \& Poole, 1996). Según Cabanach, Valle et al. (2010), el nivel de eficacia percibida en el afrontamiento se relaciona con el grado de satisfacción. Si el estudiante percibe un alto nivel de eficacia en su afrontamiento, éste condicionará sus esfuerzos a la hora de enfrentarse a los problemas y atestiguará así un grado de satisfacción mayor.

Nuestros resultados estarían en la línea de lo aportado por diversas investigaciones con alumnos universitarios (Cornejo \& Lucero, 2005; Fierro \& Jiménez, 2002; Freire et al., 2016; Thomas et al., 2017), que han concluido la existencia de una correlación positiva entre el manejo de estrategias de afrontamiento activo y un mayor sentimiento de bienestar en el estudiante. Se considera que el manejo adecuado de las estrategias de afrontamiento se relaciona íntimamente con la prevención del malestar psicológico (Kramtsova, Saarnio, Gordeeva \& Williams, 2007; Pozos-Radillo et al., 2016) y con una percepción subjetiva de bienestar (Parsons et al., 1996).

Nuestros resultados también ponen claramente de manifiesto el efecto diferencial del afrontamiento activo y el afrontamiento social en la reducción de las manifestaciones psicofisiológicas de estrés. El afrontamiento social no tiene efecto significativo en la reducción del estrés, salvo cuando se comparan los grupos de estudiantes con un bajo afrontamiento activo. Sólo en este caso parece tener un efecto compensador. Es posible que estas diferencias sean más evidentes en el ámbito en que hemos desarrollado nuestra investigación, de claro carácter competitivo y en el que la motivación de logro está más potenciada en los estudiantes.

Estos resultados también aportan información para el diseño de programas que permitan el entrenamiento en habilidades de afrontamiento y manejo del estrés por parte de los estudiantes universitarios. Dichos programas podrían resultar extremada- 
mente útiles para prevenir la aparición de estrés, contribuyendo a dotarles de recursos intrapsíquicos necesarios para afrontar con garantías las demandas académicas y el manejo del estrés (Brougham, Zail, Mendoza \& Miller, 2009; Fornés-Vives et al., 2016).

Una de las tareas de la universidad como institución es asumir el reto de mitigar el impacto del estrés académico entre sus estudiantes mediante la articulación de procedimientos de ayuda, orientación y soporte socioemocional que faciliten un afrontamiento constructivo de las exigencias académicas y minimicen los posibles efectos negativos sobre su salud y su estado de bienestar general (Denovan \& Macaskill, 2017; FornésVives et al., 2016). Manejar el estrés, fomentar los recursos de afrontamiento, promover el apoyo motivacional y la afectividad positiva, formar habilidades para saber responder a las demandas (Brougham et al., 2009; González \& Landero, 2008) parecen propuestas de obligado cumplimiento por parte de las instituciones para ayudar al estudiante a afrontar con efectividad el estrés, evitar en la medida de lo posible su vulnerabilidad frente al mismo y mejorar sus opciones de adaptación y su desempeño académico.

Resulta extremadamente llamativo encontrarnos con el hecho de que, desde las primeras propuestas para implantar programas tanto preventivos (Polo, Hernández \& Pozo, 1996) como de corte intervencionista (Felner, Gister \& Primavera, 1982; Hernández, Pozo \& Polo, 1994; Pascarella, Terenzini \& Wolfle, 1986; Tinto, 1982) en las universidades para ayudar a los estudiantes en su periodo de transición y en la adaptación a la vida universitaria y a sus exigencias, se puede constatar actualmente una carencia evidente de recursos, instrumentos y medidas que posibiliten la prevención de problemas de estas características en los estudiantes universitarios actuales (Fornés-Vives et al., 2016).

\section{Referencias}

Brougham, R. R., Zail, C. M., Mendoza, C. M., \& Miller, J. R. (2009). Stress, sex differences, and coping strategies among college students. Current Psychology, 28(2), 85-97. doi: http://dx.doi.org/10.1007/s12144-009-9047-0.

Cabanach, R. G., Cervantes, R. F., González-Doniz, L. G., \& Freire, C. (2010). Estresores académicos percibidos por estudiantes universitarios de ciencias de la salud. Fisioterapia, 32(4), 151-158. doi: http://dx.doi.org/10.1016/j.ft.2010.01.005

Cabanach, R. G., González-Millán, P., \& Freire, C. (2009). El afrontamiento del estrés en estudiantes de ciencias de la salud: diferencias entre hombre y mujeres. Aula Abierta, 37(2), 3-10. Recuperado de https://dialnet.unirioja.es/servlet/articulo?codigo=3088531

Cabanach, R. G., Valle, A., Rodríguez, S., Piñeiro, I., \& Freire, C. (2010). Escala de afrontamiento del estrés académico (A-CEA). Revista Iberoamericana de Psicología y Salud, 1(1), 51-64. Recuperado de www.redalyc.org/pdf/2451/245116411005.pdf

Carver, C. S., Scheier, M. F., \& Pozo, C. (1992). Conceptualizing the process of coping with health problems. En H. S. Friedman (Ed.), Hostility, coping, and health (pp. 167199). Washington, DC: American Psychological Association.

Compas, B. E., Connor-Smith, J. K., Saltzman, H., Thomsen, A. H., \& Wadsworth, M. E. (2001). Coping with stress during childhood and adolescence: problems, progress, and potential in theory and research. Psychological Bulletin, 127(1), 87-127. doi: http:// dx.doi.org/10.1037/0033-2909.127.1.87. 
Cornejo, M., \& Lucero, M. C. (2006). Preocupaciones vitales en estudiantes universitarios relacionado con bienestar psicológico y modalidades de afrontamiento. Fundamentos en Humanidades, 12, 143-153. Recuperado de www.redalyc.org/pdf/184/18412608.pdf

de la Fuente, J., Fernández-Cabezas, M., Cambil, M., Vera, M. M., González-Torres, M. C., \& Artuch-Garde, R. (2017). Linear relationship between resilience, learning approaches, and coping strategies to predict achievement in undergraduate students. Frontiers in Psychology, 8, 1039. doi: https://doi.org/10.3389/fpsyg.2017.0103.

Denovan, A., \& Macaskill, A. (2017). Stress and subjective well-being among first year UK undergraduate students. Journal of Happiness Studies, 18(2), 505-525. doi: https:// doi.org/10.1007/s10902-016-9736-y.

Felner, R. D., Gister. M., \& Primavera, J. (1982). Primary prevention during school transition: Social support and environmental structure. American Journal of Community Psychology, 10(3), 277-290. doi: http://dx.doi.org/10.1007/BF00896495.

Fernández, C., \& Polo, M. T. (2011). Afrontamiento, estrés y bienestar psicológico en estudiantes de educación social de nuevo ingreso. EduPsykhé: Revista de Psicología y Psicopedagogía, 10(2), 177-192. Recuperado de https://dialnet.unirioja.es/servlet/ articulo? codigo $=3896794$

Fierro, C., \& Jiménez, J. A. (2002). Bienestar, variables personales y afrontamiento en jóvenes universitarios. Escritos de Psicología, 6, 85-91. Recuperado de http://www. escritosdepsicologia.es/esp/numanteriores/num6.html

Fornés-Vives, J., Garcia-Banda, G., Frias-Navarro, D., \& Rosales-Viladrich, G. (2016). Coping, stress, and personality in Spanish nursing students: A longitudinal study. Nurse Education Today, 36, 318-323. doi: https://doi.org/10.1016/j.nedt.2015.08.011.

Freire, C., Ferradás, M. D. M., Valle, A., Núñez, J. C., \& Vallejo, G. (2016). Profiles of psychological well-being and coping strategies among university students. Frontiers in Psychology, 7, 1554. doi: http://dx.doi.org/10.3389/fpsyg.2016.01554

González, M. T., \& Landero, R. (2008). Confirmación de un modelo explicativo del estrés y de los síntomas psicosomáticos mediante ecuaciones estructurales. Revista Panamericana de Salud Pública, 23(1), 7-18. Recuperado de https://www.scielosp.org/ pdf/rpsp/v23n1/a02v23n1.pdf

Hair, J. E., Anderson, R. E., Tatham, R. L., \& Black W. C. (1999). Multivariate data analysis (6 $6^{\mathrm{a}}$ ed.). Upper Saddle River, NJ: Prentice-Hall.

Hernández, J. M., Pozo, C., \& Polo, A. (1994). Ansiedad ante los exámenes: un programa para su afrontamiento de forma eficaz. Valencia: Promolibro.

Johnson, D., Jhonson, R. (2002). Teaching Students How to Cope with adversity: the Three Cs. En Frydenberg, E. (Comp.), Beyond Coping. Meeting goals, visions, and challenges. New York: Oxford University Press

Khramtsova, I., Saarnio, D. A., Gordeeva, T., \& Williams, K. (2007). Happiness, life satisfaction and depression in college students: Relations with student behaviours and attitudes. American Journal of Psychological Research, 3(1), 8-16. Recuperado de https://www.mcneese.edu/f/c/9ac70696/ajpr02_07.pdf

Montero, I., \& León, O. G. (2002). Clasificación y descripción de las metodologías de investigación en Psicología. International Journal of Clinical and Health Psychology, 2(3), 503-508. Recuperado de www.aepc.es/ijchp/articulos_pdf/ijchp-53.pdf 
Parsons, A., Frydenberg, E., \& Poole, C. (1996). Overachievement and coping strategies in adolescent males. British Journal of Educational Psychology, 66(1), 109-114. doi: http://dx.doi.org/10.1111/j.2044-8279.1996.tb01180.x.

Pascarella, E. T., Terenzini, P. T., \& Wolfle, L. M. (1986). Orientation to college and freshman year persistence/withdrawal decisions. The Journal of Higher Education, 57(2), 155-175. Recuperado de https://www.jstor.org/stable/1981479

Pastor, D. A., Barron, K. E., Miller, B. J., \& Davis, S. L. (2007). A latent profile analysis of college student's achievement goal orientation. Contemporary Educational Psychology, 32(1), 8-47. doi: http://dx.doi.org/10.1016/j.cedpsych.2006.10.003.

Polo, A., Hernández, J. M. , \& Pozo, C.(1996). Evaluación del estrés académico en estudiantes universitarios. Ansiedad y estrés, 2(2), 159-172. Recuperado de https:// dialnet.unirioja.es/servlet/articulo? codigo $=186664$

Pozos-Radillo, E., Preciado-Serrano, L., Plascencia-Campos, A., Valdez-López, R., \& Morales-Fernández, A. (2016). Psychophysiological manifestations associated with stress in students of a public university in Mexico. Journal of Child and Adolescent Psychiatric Nursing, 29(2), 79-84. doi: http://dx.doi.org/10.1111/jcap.12142.

Somerfield, M. R., \& McCrae, R. R. (2000). Stress and coping research: Methodological challenges, theoretical advances, and clinical applications. American Psychologist, 55(6), 620-625. doi: http://dx.doi.org/10.1037/0003-066X.55.6.620.

Thomas, C. L., Cassady, J. C., \& Heller, M. L. (2017). The influence of emotional intelligence, cognitive test anxiety, and coping strategies on undergraduate academic performance. Learning and Individual Differences, 55, 40-48. doi: https:// doi.org/10.1016/j.lindif.2017.03.001.

Tinto, V. (1982). Defining dropout: A matter of perspective. En E. T. Pascarella (Ed.), Studying student attrition (pp. 3-15). San Francisco, CA: Jossey-Bass.

Zeidner, M., \& Saklofske, D. (1996). Adaptative and maladaptative coping. En M. Zeidner \& N. S. Endler (Eds.), Handbook of coping: Theory, research, applications (pp. 505-531). New York: Wiley.

Fecha de recepción: 5 de abril de 2017

Fecha de revisión: 15 de abril de 2017

Fecha de aceptación: 22 de marzo de 2018 
